\title{
Structure of characteristic Lyapunov vectors in spatiotemporal chaos
}

\author{
Diego Pazó, ${ }^{1, *}$ Ivan G. Szendro, ${ }^{1,2, \dagger}$ Juan M. López, ${ }^{1,+}$ and Miguel A. Rodríguez ${ }^{1, \S}$ \\ ${ }^{1}$ Instituto de Física de Cantabria (IFCA), CSIC-Universidad de Cantabria, E-39005 Santander, Spain \\ ${ }^{2}$ Departamento de Física Moderna, Universidad de Cantabria, Avenida Los Castros, E-39005 Santander, Spain
}

(Received 22 April 2008; published 17 July 2008)

\begin{abstract}
We study Lyapunov vectors (LVs) corresponding to the largest Lyapunov exponents in systems with spatiotemporal chaos. We focus on characteristic LVs and compare the results with backward LVs obtained via successive Gram-Schmidt orthonormalizations. Systems of a very different nature such as coupled-map lattices and the (continuous-time) Lorenz '96 model exhibit the same features in quantitative and qualitative terms. Additionally, we propose a minimal stochastic model that reproduces the results for chaotic systems. Our work supports the claims about universality of our earlier results [I. G. Szendro et al., Phys. Rev. E 76, 025202(R) (2007)] for a specific coupled-map lattice.
\end{abstract}

DOI: 10.1103/PhysRevE.78.016209

PACS number(s): 05.45.Jn, 05.40.-a, 05.45.Ra

\section{INTRODUCTION}

Nonlinear spatially extended systems often exhibit spatiotemporal chaos (STC), i.e., an apparent randomness in both space and time. Lyapunov exponents (LEs) measure the exponential separation (or convergence) of nearby trajectories and provide an important tool to characterize chaos in nonlinear dynamical systems [1-3]. Not only exponential separation rates but also the associated directions in tangent space, the so-called Lyapunov vectors (LVs), are required when trying to tackle many important aspects of STC, such as, for instance, the role of hydrodynamic modes [4], extensivity properties [5], or predictability questions [2], among others. Random initial errors evolve in time and asymptotically align with the main LV corresponding to the most unstable direction. In practice, this limit is reached exponentially fast, so the memory of the initial perturbation is quickly lost.

In extended systems, the spatial distribution and correlations of LVs are crucial to deal with questions such as predictability [6]. The relevance of spatial correlations is particularly apparent in the context of weather forecasting (see, for instance, [7]).

Localization of LVs in several distributed systems has been noticed and discussed in some extent in the literature [8-15]. This phenomenon has been termed dynamical localization of errors [16]: The main LV rapidly tends to concentrate around a narrow region of space. In homogenous systems, where all degrees of freedom are equivalent, the localization locus is not anchored to any fixed site, but keeps moving all over the system. However, in the case of inhomogeneous systems LVs become strongly localized at certain fixed pinning centers and the phenomenon can be understood in terms of the problem of diffusion in quenched random potentials [17].

\footnotetext{
*pazo@ifca.unican.es

†szendro@ifca.unican.es; Present address: Max Planck Institute for the Physics of Complex Systems, Nöthnitzer Straße 38, 01187 Dresden, Germany.

†lopez@ifca.unican.es

§odrigma@ifca.unican.es
}

Recently, the evolution of infinitesimal perturbations in spatially extended chaotic systems has been shown to be generically described by Langevin-type equations with multiplicative noise [12,16-19]. A remarkable observation in many systems [16] is that, after a suitable logarithmic transformation, the statistical description of the dynamics of perturbations is captured by the prototypical stochastic surface growth equation of Kardar-Parisi-Zhang (KPZ) [20]. In the surface picture, erratic fluctuations, due to the chaotic nature of the trajectory, are treated as an effective noise. The existence of short-range correlations, coming from the deterministic nature of the trajectory, are irrelevant for the scaling description of the surface statistics. It is only natural that the existence of long-range correlations [21] or a fat tail noise [19] may change the universality class observed. The surface picture has also been shown to be very useful to deal with the dynamics of finite perturbations in the presence of STC $[6,18,22]$.

In view of the successful description of the main LV as a nonequilibrium rough surface, a question that naturally arises is, to what extent can we describe LVs corresponding to other unstable directions in terms of surface roughening processes? This is precisely the question we recently addressed in a Rapid Communication [23], and that we develop here for a variety of systems.

In the existing literature, one finds that the LVs are commonly defined as the vectors that appear as a byproduct of the standard Gram-Schmidt orthonormalization procedure to obtain the LEs. This is largely due to the popularity of Benettin's algorithm $[24,25]$ to compute the Lyapunov spectrum in all kinds of dynamical systems. However, these vectors do not point in the most unstable directions, but are forced to form an orthogonal set. This is not a minor point because these vectors lack the intended physical meaning, which ultimately renders the Gram-Schmidt vectors useless for many purposes. For example, when the $n$th Gram-Schmidt LV, $\mathbf{e}_{n}(t)$, is left to evolve freely, it will not grow exponentially with its associated LE $\lambda_{n}$ (apart from the case $n=1$ ); instead, $e_{n}(t)$ will generally collapse in the direction of the first LV. However, if the same vector $e_{n}(t)$ is integrated backwards in time, it will shrink with exponent $-\lambda_{n}$ (neglecting numerical round-off errors). Not less important is the fact that Gram- 
Schmidt LVs depend on the scalar product convention used because it defines the orthogonalization condition.

These important caveats have attracted renewed interest in the problem of finding the correct set of vectors that carry the dynamical information in systems exhibiting STC. Recent work has focused on the properties of a different set of vectors $[23,26,27]$, the characteristic LVs (also called covariant LVs), that are invariant under time reversal and covariant with the dynamics. This vector set is independent of the scalar product used and provides an intrinsic decomposition in tangent space, which should correspond exactly with Oseledec's splitting [1]. Although the existence of the characteristic LVs has long been known [1,28-30], it was not until recently that efficient algorithms were devised to compute them $[26,27]$.

We have recently shown in Ref. [23] that characteristic LVs carry important information about the real-space structure, localization properties, and space-time correlations, which can be put in the form of a dynamical scaling of the associated rough surfaces. These scaling properties were demonstrated for the particular case of lattices of coupled logistic maps, but conjectured to be valid for a wide range of systems (at least including all those reported in Ref. [16] as belonging to the KPZ class).

In this paper, we study the spatiotemporal structure of the characteristic LVs in different model systems exhibiting STC. Our aim is to analyze the spatial structure of the characteristic LVs. In particular, we wish to provide further verification of the previously reported scale-invariant properties of the LV surfaces (to be defined below) and its validity for systems that differ significatively from the special case of coupled-map lattices. Here we analyze systems of a very different nature, including a coupled-map lattice (CML), the (continuous-time) Lorenz '96 model, and a minimal stochastic partial differential equation (PDE). We show that the leading LVs (corresponding to the largest LEs) generically exhibit scale-invariant properties inherited from those of the main vector. Our present results confirm and extend our earlier claim [23] concerning the generic, model-independent scaling properties of characteristic LVs corresponding to unstable intrinsic directions.

\section{MODELS OF SPATIOTEMPORAL CHAOS}

Three spatially extended systems are studied in this paper: a coupled-map lattice, a continuous-time model, and a stochastic equation. These models cover a range of dynamical systems of very different nature, including discrete and continuous systems. We study models that exhibit STC for a range of parameters. Since the scaling properties that we are interested in are independent of microscopic details, our intention here is not to be exhaustive in the exploration of model parameters or different terms in a particular model, which have no effect on the scaling properties whatsoever. On the contrary, our aim is to address much more generic types of models, such as those that are discrete or continuous in space or time.

\section{A. Coupled-map lattices}

Coupled-map lattices are simple prototypes of STC at low computational cost [2]. This ultimately explains their wide-

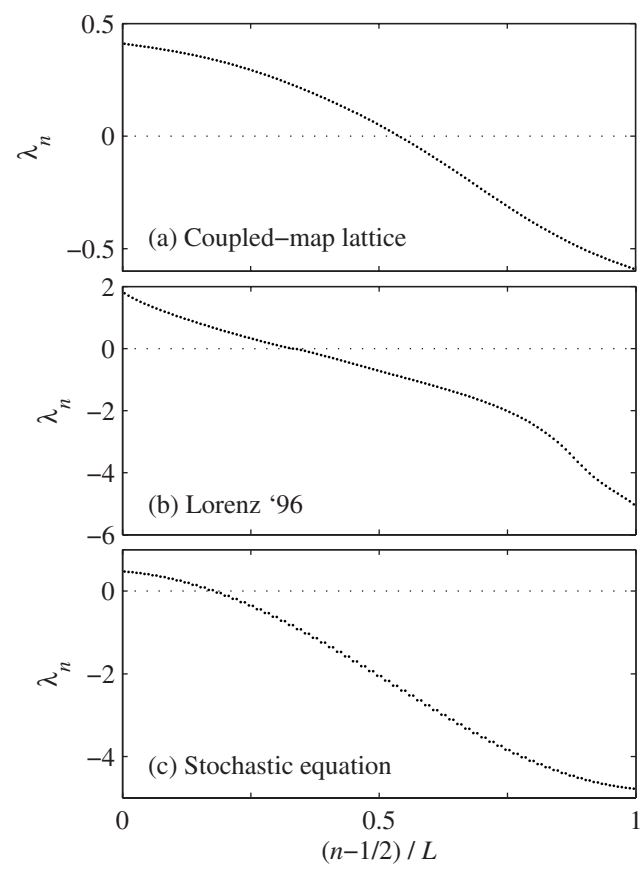

FIG. 1. Lyapunov spectra for the three models studied in this paper. We follow the standard convention and sort LEs in decreasing order $\lambda_{1} \geqslant \lambda_{2} \geqslant \cdots \geqslant \lambda_{L}$. (The LEs were obtained for $L=128$; larger systems yield Lyapunov spectra that overlap these ones.)

spread use to study different aspects of STC, which would be prohibitively demanding in computation time should PDEs be used, for instance. Here, we consider a ring of $L$ maps with diffusive coupling,

$$
u_{i}(t+1)=\epsilon f\left[u_{i+1}(t)\right]+\epsilon f\left[u_{i-1}(t)\right]+(1-2 \epsilon) f\left[u_{i}(t)\right],
$$

where $\epsilon$ is the coupling parameter and $f$ is a map with chaotic dynamics. Infinitesimal random perturbations evolve in tangent space following the linear equation

$$
\begin{aligned}
\delta u_{i}(t+1)= & \epsilon f^{\prime}\left[u_{i+1}(t)\right] \delta u_{i+1}(t)+\epsilon f^{\prime}\left[u_{i-1}(t)\right] \delta u_{i-1}(t) \\
& +(1-2 \epsilon) f^{\prime}\left[u_{i}(t)\right] \delta u_{i}(t),
\end{aligned}
$$

where $f^{\prime}(\varrho)$ is just the derivative of the map $f(\varrho)$ with respect to its argument $\varrho$. We have recently reported in Ref. [23] about our study of the space-time structure of LVs in the case of the lattice of coupled logistic maps $f(\varrho)=4 \varrho(1-\varrho)$. Here, as a further example we include the study of a different type of map. The results we report on in this paper (see below) are analogous to those obtained for logistic maps, and thus we may conclude that no important differences should arise among one-dimensional CMLs composed of continuous chaotic maps in one variable. Throughout this paper, we consider the skew tent map with the same parameters as those in Ref. [27],

$$
f(\varrho)=\left\{\begin{array}{cc}
a \varrho & (\varrho \leqslant 1 / a) \\
a(\varrho-1) /(1-a) & (\varrho>1 / a)
\end{array}\right.
$$

with $a=2.3$. The Lyapunov spectrum of the CML in Eq. (1) for a coupling strength $\epsilon=0.2$ is shown in Fig. 1(a). 


\section{B. Lorenz '96 model}

The second model we consider in this paper is an example of a chaotic continuous-time system. This model is in many aspects very different from a CML model due to the continuous character of the time variable. We study the model proposed by Lorenz in 1996 [31] as a toy model in the context of weather dynamics. We consider the variables $y_{i}$ defined in a ring, $i=1, \ldots, L$, and the evolution equations

$$
\frac{d}{d t} y_{i}=-y_{i}-y_{i-1}\left(y_{i-2}-y_{i+1}\right)+F \text {. }
$$

The variables $y_{i}$ may be looked at as the values of some unspecified scalar meteorological observable, like a vorticity or temperature, at equally spaced sites extending around a latitude circle [32]. The model contains quadratic, linear, and constant terms mimicking advection, dissipation, and external forcing, respectively.

An infinitesimal perturbation $\delta y_{i}(t)$ evolves in tangent space according to the linearized dynamics,

$$
\frac{d}{d t} \delta y_{i}=-\delta y_{i}-\left(y_{i-2}-y_{i+1}\right) \delta y_{i-1}-y_{i-1} \delta y_{i-2}+y_{i-1} \delta y_{i+1},
$$

which also governs the dynamics of any characteristic LV, as they are freely evolving covariant perturbations.

Regardless of how well or poor Eq. (4) represents the atmosphere, the model is nowadays an essential tool in studies of weather dynamics as a testbed for forecasting techniques like breeding or singular vectors [31-33]. For $F=8$, the model exhibits STC, as demonstrated by computing the Lyapunov spectrum shown in Fig. 1(b). A Runge-Kutta scheme is usually recommended for the numerical integration of Eq. (4) to avoid numerical instabilities. We have used a fourth-order Runge-Kutta integration algorithm with time step $\Delta t=10^{-2}$, while to achieve the same precision with the Euler method a much smaller time step, $\Delta t=1.5 \times 10^{-4}$, was needed.

\section{Multiplicative stochastic equation}

The third model we study is a multiplicative stochastic equation, which mimics the linear evolution of infinitesimal perturbations in tangent space for spatio-temporal chaotic systems. Pikovsky and Politi proposed [16] this stochastic PDE as the proper candidate for modeling the statistical features of the dynamics of freely evolving perturbations. Therefore, the analysis of this model will show to what extent the observed scaling of characteristic LV surfaces is generic and model-independent in the context of STC.

We consider a perturbation $\phi(x, t)$, initially homogeneous and random, whose time evolution can be described, in a statistical sense, by the multiplicative Langevin equation

$$
\partial_{t} \phi=\zeta(x, t) \phi+\partial_{x x} \phi,
$$

where $\zeta$ is a noise term that accounts for the chaotic fluctuations and one can simply assume it to be Gaussian and uncorrelated: $\left\langle\zeta(x, t) \zeta\left(x^{\prime}, t^{\prime}\right)\right\rangle=2 \sigma \delta\left(x-x^{\prime}\right) \delta\left(t-t^{\prime}\right)$. It is worth stressing here that the presence of short-range correlations in the noise term $\zeta$ (due to the purely deterministic nature of the fluctuations) is actually irrelevant for the statistical description in the long-wavelength limit, as already shown in the original work of Pikovsky and Politi [16].

We have numerically integrated Eq. (6) by a stochastic Euler scheme (the noise term up to order $\Delta t$ ) with a space and time step $\Delta x=1$ and $\Delta t=10^{-2}$. The Lyapunov spectrum was computed and averaged over different noise realizations (equivalent to different trajectories). In Fig. 1(c), we plot the LEs for a noise amplitude $\sigma=0.5$.

The multiplicative Langevin equation (6) can be seen as a stochastic field theory for the evolution of random errors in extended homogeneous systems. This stochastic model has been found to describe the statistical properties of perturbations in many dynamical systems ranging from lattices of logistic, tent, or symplectic maps to the complex GinzburgLandau equation $[12,16,21]$. It has also been extended to construct a stochastic field theory of chaotic synchronization of extended systems [34,35]. Very recently, it has also been shown that a version of Eq. (6), which includes quenched disorder terms, describes the propagation of perturbations in inhomogeneous chaotic systems [17].

Interestingly, the application of the Hopf-Cole transformation, $h=\ln |\phi|$, immediately maps the problem into the KPZ equation for surface growth,

$$
\partial_{t} h=\zeta+\left(\partial_{x} h\right)^{2}+\partial_{x x} h
$$

which ultimately justifies why the log-transformed (main) Lyapunov vector of many spatiotemporal chaotic systems is found to belong to the KPZ universality class $[12,16]$.

There is an interesting caveat concerning this mapping, which has not been noticed before in the context of STC. One can see that Eq. (6) is invariant under the sign change of the field $\phi \rightarrow-\phi$. However, the solutions of Eq. (6) actually exhibit a spontaneous breaking of this essential symmetry. In our numerical integration, we observe that for any random initial condition, no matter the spatial distribution of signs for the initial field $\phi(x, t=0)$, with probability 1 , the solutions of Eq. (6) asymptotically become either strictly positive or negative [i.e., for long enough times $\phi(x, t) \neq 0$ for all $x]$. The reason for this symmetry breaking can be traced back to the mathematical properties of Eq. (6). The key observation is that the dynamics governed by Eq. (6) cannot produce new zeros of the field $\phi$. Therefore, sites where $\phi$ changes sign can only diffuse in the $x$ axis and, in the event two $\phi$-zeros collide, disappear. As we will see later on, the annihilation of zeros is crucial to understand the spatial structure of characteristic LVs.

\section{LYAPUNOV VECTORS}

In short, LVs are defined as the vectors in tangent space that point toward the directions such that a given perturbation expands (shrinks) with the corresponding LE. Their physical significance arises from Oseledec's theorem [36]. Let us now discuss the definition and physical meaning of backward, forward, and characteristic LVs. 
Consider a nonlinear dynamical system governed by

$$
\frac{d}{d t} \boldsymbol{u}(t)=\mathbf{Y}[\boldsymbol{u}],
$$

where $\boldsymbol{u}(t) \in \mathbb{R}^{L}$ is the position of the system in phase space and $\mathbf{Y}: \mathbb{R}^{L} \rightarrow \mathbb{R}^{L}$ is the nonlinear evolution operator. Infinitesimal perturbations $\boldsymbol{\delta} \boldsymbol{u}(t)$ follow the linear dynamics given by the tangent space equations,

$$
\frac{d}{d t} \boldsymbol{\delta} \boldsymbol{u}(t)=\frac{\partial \mathbf{Y}[\boldsymbol{u}]}{\partial \boldsymbol{u}} \boldsymbol{\delta} \boldsymbol{u}(t),
$$

which implies that the perturbation can be computed at time $t$ from the perturbation at an earlier time $t_{0}$ as

$$
\boldsymbol{\delta} \boldsymbol{u}(t)=\mathbf{M}\left(t, t_{0}\right) \boldsymbol{\delta} \boldsymbol{u}\left(t_{0}\right),
$$

where $\mathbf{M}\left(t_{0}, t\right)=\mathbf{M}\left(t, t_{0}\right)^{-1}$ is some linear operator.

\section{A. Backward (and forward) Lyapunov vectors}

According to Oseledec's theorem [36] (details can also be found in Ref. [1]), there exists the remote past limit symmetric operator $\quad \boldsymbol{\Phi}_{\mathrm{b}}(t)=\lim _{t_{0} \rightarrow-\infty}\left[\mathbf{M}\left(t, t_{0}\right) \mathbf{M}^{*}\left(t, t_{0}\right)\right]^{1 /\left[2\left(t-t_{0}\right)\right]}$, where $\mathbf{M}^{*}$ is the adjoint operator. All $L$ eigenvalues of $\boldsymbol{\Phi}_{\mathrm{b}}(t)$ are positive time-independent numbers that can be written as $\exp \left(\lambda_{n}\right)$, where $\lambda_{n}$ are the LEs, and the corresponding eigenvectors form an orthonormal basis $\left\{\mathbf{e}_{n}(t)\right\}, n=1, \ldots, L$. These eigenvectors are called backward LVs [28] and represent the directions in tangent space that, at the present time $t$, are seen to have grown at exponential rates $\lambda_{n}$ since the remote past. The set of backward LVs is precisely the orthonormal set obtained using the standard Gram-Schmidt orthogonalization method to compute the LEs [37].

Conversely, forward LVs form a different orthonormal set of vectors analogous to backward LVs, but with the temporal properties inverted. In this case, they are obtained as the eigenvectors of the far future limit operator $\lim _{t_{0} \rightarrow \infty}\left[\mathbf{M}^{*}\left(t_{0}, t\right) \mathbf{M}\left(t_{0}, t\right)\right]^{1 /\left[2\left(t_{0}-t\right)\right]}$, which obviously has the same eigenvalues as $\boldsymbol{\Phi}_{\mathrm{b}}(t)$. When left to evolve freely from the present time $t$, the $n$th forward LV grows exponentially in the far future at a rate given by the corresponding LE $\lambda_{n}$. However, under reverse (time backwards) integration, all forward LVs collapse into the last forward LV.

The popularity of the algorithm of Benettin et al. [24,25] for computing the first $n$ Lyapunov exponents, via successive Gram-Schmidt orthonormalization of a set of $n$ vectors that evolve according to the linear equations in tangent space, has caused many authors to consider using the resulting orthonormal set $\left\{\mathbf{e}_{n}(t)\right\}, n=1, \ldots, L$ as the Lyapunov vectors. As mentioned in the Introduction, the use of this set of vectors poses serious problems in certain applications. Any of the $L$ backward LV tends to align exponentially fast with the first LV. This has to be avoided by the externally imposed orthogonalization, which "resets" the vector set every few time steps. Moreover, different scalar products produce different sets of backward and forward LVs.

\section{B. Characteristic Lyapunov vectors}

In order to construct a complete set of $L$ characteristic (or covariant) vectors, $\left\{\mathbf{g}_{n}(t)\right\}, n=1, \ldots, L$, independent of the scalar product and having the wanted topological properties, one has to intersect the subspaces spanned by the backward and forward LV in a precise manner as discussed by Eckmann and Ruelle [1].

At variance with backward and forward LVs, characteristic vectors have the desired topological and dynamical properties: (i) They are independent of the scalar product; (ii) they reduce to the Floquet eigenvectors for a periodic orbit [30]; (iii) any given $\boldsymbol{g}_{n}(t)$ grows at an exponential rate given by the associated LE $\lambda_{n}$ in the far future, and with rate $-\lambda_{n}$ backward-integrating to the remote past (under the linearized equations in tangent space, with no orthogonalization or any other external constraint). For instance, in chaotic continuous-time systems, and in contrast with backward LVs, there is a characteristic LV tangent to the trajectory that corresponds to the zero LE associated with time-shift invariance.

Contrary to the (artificial) orthogonal disposition of backward LVs, characteristic LVs generally do not form an orthogonal set. We note that the first backward and characteristic LVs are tangent, $\boldsymbol{g}_{1}(t) \propto \boldsymbol{e}_{1}(t)$. For $n>1$, the $n$th characteristic LV is a linear combination of backward LVs from 1 to $n$.

Although Eckmann and Ruelle [1] already discussed these ideas in 1985, they had received little attention in the literature until very recently. This is partially due to the fact that implementing such a theoretical construction is by no means a simple task from a computational point of view. Only very recently, Wolfe and Samelson [26] proposed a computationally efficient algorithm to obtain the set of characteristic LVs. We have used this algorithm in all our calculations, and technical details can be found in the Appendix. Also, Ginelli et al. proposed a similar algorithm [27].

In the rest of this paper, we study the spatial structure of LVs, focusing on universal features that are shared among different models of STC.

\section{SURFACE GROWTH PICTURE}

In systems with spatiotemporal chaos, the first LV localizes in space, so that its magnitude spans several orders of magnitude between the top and the valleys. In homogeneous systems, translational invariance implies that the localization site is not static.

It was recognized some time ago $[12,16]$ that the spatiotemporal dynamics of the first $\mathrm{LV}$ is much more understandable as a surface to be obtained after Hopf-Cole transforming the vector. Until recently, very little was known about the spatial correlations of characteristic (or backward) vectors for $n>1$. We have reported [23] on the existence of intrinsic length scales and have determined the form of the spatiotemporal correlations of LVs corresponding to the leading unstable directions by translating the problem to the language of scale-invariant growing surfaces. We found that characteristic LVs corresponding to the most unstable directions also exhibit spatial localization, strong clustering around given spatiotemporal loci, and remarkable dynamic scaling properties of the corresponding surfaces. In contrast, any two backward LVs localize in different sites since they 

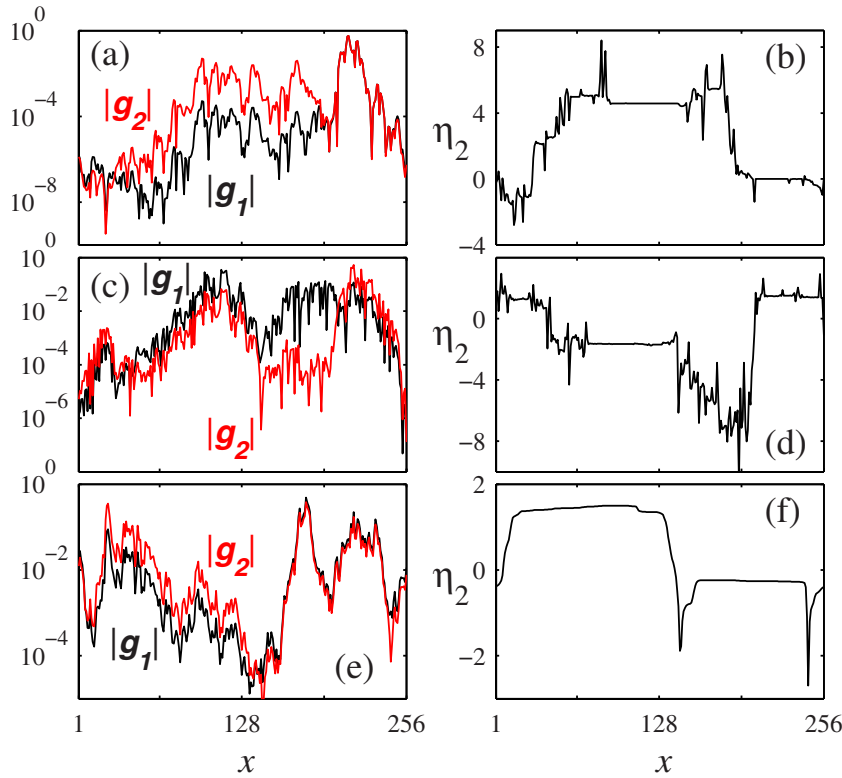

FIG. 2. (Color online) The first and the second characteristic Lyapunov vectors for (a) coupled-map lattice (1), (c) Lorenz '96 model, and (e) Stochastic equation (6). Note that we take the absolute values and a logarithmic scale for the $y$ axis. Panels (b), (d), and (f) show the fields $\eta_{2}=h_{2}-h_{1}$ for the three models.

are mutually orthogonal. Also, they do not exhibit dynamic scaling due to artifacts in the dynamical correlations by construction [23]. Our results were based on numerical studies of lattices of coupled maps, but conjectured to be generically valid for a wide range of systems. Our aim here is to extend our previous analysis and put it in a wider context. For this purpose, the analysis of the stochastic model Eq. (6) has a particular significance.

Figures 2(a), 2(c), and 2(e) show typical snapshots of the first and second characteristic LVs in logarithmic scale for the three models introduced in Sec. II. One can see that both vectors may localize in the same site (which is not possible for backward LVs due to their mutual orthogonality). For every characteristic $\mathrm{LV}, \boldsymbol{g}_{n}(t)=\left[g_{n}(x, t)\right]_{x=1}^{x=L}$, we define a surface via the Hopf-Cole transformation, $h_{n}(x, t)=\ln \left|g_{n}(x, t)\right|$. For the sake of comparison, we will also consider the surfaces associated with backward LVs: $h_{n}(x, t)=\ln \left|e_{n}(x, t)\right|$. After the mapping, the $n$th LE corresponds to the average velocity of the corresponding $n$th surface, $\left\langle(1 / L) \sum_{x=1}^{x=L} h_{n}(x, t)\right\rangle$ $=\left\langle\ln \prod_{x=1}^{x=L}\left|g_{n}(x, t)\right|^{1 / L}\right\rangle \approx \lambda_{n} t$.

The surface growth formalism has allowed us to identify different universality classes in spatiotemporal chaotic systems $[12,16,19,21]$. In particular, the universality class of KPZ has been widely observed in non-Hamiltonian systems with no special conservation laws, discontinuities, or broken symmetries. This includes, among others, lattices of coupled logistic maps, and the Ginzburg-Landau and KuramotoSivashinsky PDEs. The three model systems studied in this paper (see Sec. II) also belong to KPZ universality class. This can be confirmed by calculating the so-called dynamic and roughness exponents.

Interestingly, we have found that the $n$th LV (either characteristic or backward) is a piecewise copy of the main LV.
This behavior is conveniently captured by the differencefield $\eta_{n} \equiv h_{n}-h_{1}$. For instance, in Figs. 2(b), 2(d), and 2(f) we plot the difference-field $\eta_{2}=h_{2}-h_{1}$, which reveals the existence of flat regions indicating that the first and the second LV surfaces are strongly correlated. The second LV surface is loosely speaking "piecewise KPZ," since it is made of pieces that differ from the main vector at only a few sites. Actually, the $n$th LV also exhibits the same structure for increasing $n$, namely, the difference-field $\eta_{n}$ is also formed by flat regions separated by fluctuating edges. The typical plateau length of the field $\eta_{n}$ naturally defines a characteristic length scale $\ell_{n}$, below which the $n$th surface is identical to the first surface. This characteristic plateau size decreases with increasing $n$. So that, beyond some $n_{\max }$, the number of fluctuating edges is so large $\left(\ell_{n} \rightarrow 1\right.$ for $\left.n \gg n_{\max }\right)$ that the "piecewise KPZ" picture is not useful any longer.

It is remarkable that, for systems whose first LV belongs to the KPZ class, there is a finite part of the Lyapunov spectrum $\left(\lambda_{n}\right.$ with $\left.n<n_{\max }\right)$ that can be understood in terms of piecewise copies of the first vector. Note that this peculiar spatial structure can only be easily identified after the logarithmic transform. Last but not least, this spatial structure is also consistent with the fact that any characteristic LV is governed by the same tangent dynamics for a given system, Eqs. (2), (5), and (6), and a given trajectory.

\section{SPATIAL STRUCTURE}

In this section, we carry out a quantitative description of the spatial correlations of the LV surfaces $h_{n}(x, t)$. We compute the stationary structure factor $S_{n}(k)$ $=\lim _{t \rightarrow \infty}\left\langle\hat{h}_{n}(k, t) \hat{h}_{n}(-k, t)\right\rangle, \quad$ where $\quad \hat{h}_{n}(k, t)=\Sigma_{x} \exp (i k x)$ $\times h_{n}(x, t)$, and the brackets indicate an average over different system trajectories (or noise realizations in the case of the purely stochastic model). As expected, the first LV surface correlations decay as $k^{-2}$ (Fig. 3), in agreement with the KPZ universality class $[16,20]$. Interestingly, the $n$th LV surface for $n>1$ also shows scale-invariant correlations $\sim k^{-2}$, with a crossover to a different scaling regime at a wave number $k_{n}$ that depends on $n$. It is natural to link this crossover length scale to the plateaus discussed in the preceding section. Indeed, we have shown in Ref. [23] that this crossover wavelength is related to the typical size of plateaus $\mathrm{k}_{n} \sim \ell_{n}^{-1}$.

At long wavelengths, correlations of LV surfaces associated with backward and characteristic LVs decay approximately as $k^{-1}$ and $k^{-1.2}$, respectively (Fig. 3). This $1 / k$ divergence indicates extremely weak long-range spatial correlations for both classes of LVs. However, backward and characteristic vectors exhibit markedly different dynamical properties. To be precise, the imposition of orthogonality causes the mapping of a backward LV at $t$ into itself at $t$ $+\Delta t$ to convey $1 / k$ long-range correlations. On the contrary, characteristic LVs show increasing correlation lengths as time evolves, as one would expect for a surface evolving with local equations. In this case (and in contrast with backward LVs), surface correlations are found to satisfy dynamic scaling akin to growing surfaces (cf. Fig. 4 in [23]).

Deterministic equations (1) and (4) yield LVs whose spatial structure is analogous to the structure of LVs obtained 

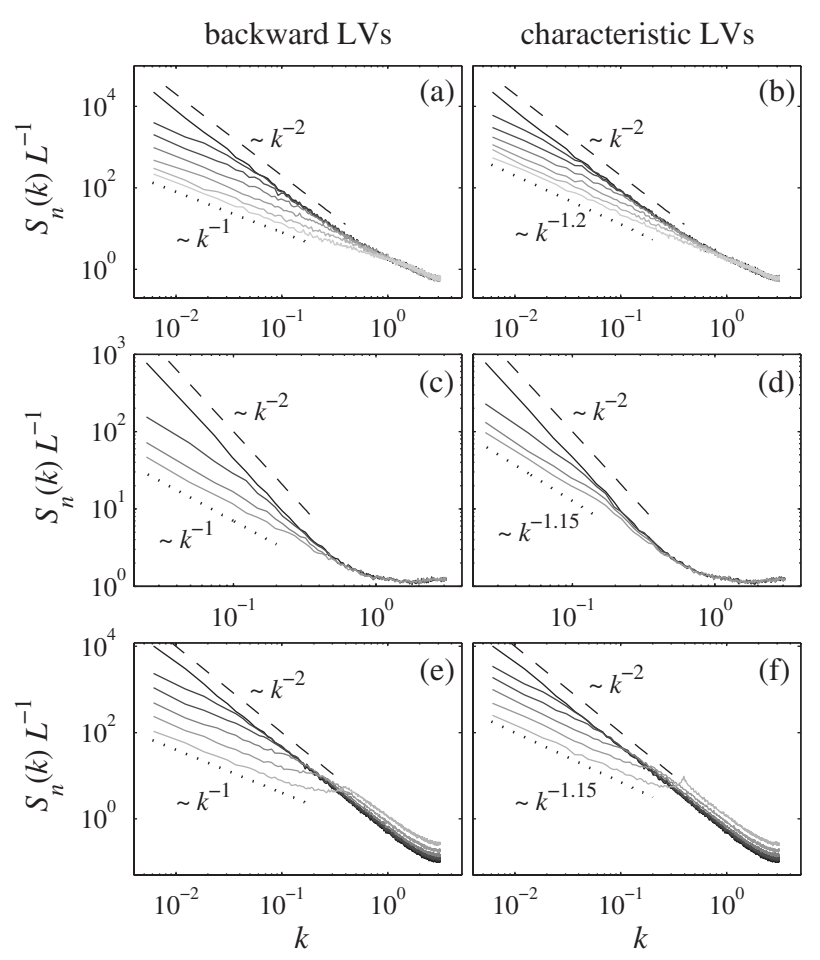

FIG. 3. Structure factors for LVs of the three models considered in this paper: (a,b) Coupled-map lattice (1) with $L=1024$, from top to bottom $n=1,4,8,16,32,64,128$; (c,d) Lorenz '96 model (4) with $L=256, n=1,4,8,12$; and (e,f) stochastic equation (6) with $L=1024, n=1,4,8,16,32,64$. In all cases, backward LVs (a,c,e) beyond the first one decay at small wavelengths as $k^{-1}$. Characteristic LVs (b,d,f) display stronger correlations with $k^{-\gamma}(\gamma$ $\approx 1.15-1.2$ ). We averaged over 200 realizations for the CML and 1000 for the other two models.

with the stochastic equation (6) with white noise. This indicates that in spatiotemporal chaotic systems of the KPZ universality class, the role of spatio-temporal correlations is insignificant in what concerns the statistical (long-time and large-scale) structure of LVs.

Finally, we recall that when $n$ becomes large $\left(n>n_{\max }\right)$, specific features of each model will show up. For instance, in the case of the multiplicative stochastic equation, the $n$th LV appears as a noisy sinusoidal function because diffusion prevails over the stochastic term. Accordingly a peak appears at intermediate wave numbers in the structure factor [see the curve for $n=64$ in Fig. 3(f)].

\section{MULTIPLICATIVE STOCHASTIC EQUATION}

The multiplicative Langevin model discussed in Sec. II C constitutes a minimal model for describing the dynamics of free perturbations in a (wide) family of systems exhibiting spatiotemporal chaos [16]. In particular, since random free perturbations rapidly tend to be tangent to the main LV, Eq. (6) also describes the scaling behavior of the first LV. As we have discussed in the preceding section, characteristic LVs are freely propagating perturbations, covariant with the dynamics as well as with the time inverted dynamics. Therefore, we conjecture here that the multiplicative stochastic model should also describe the statistics and scaling behavior of the $n$th characteristic LV, at least for $n<n_{\max }$. In this regard, the scaling properties of the $n$th characteristic LV in systems with STC should be generically linked to those of the solutions supported by the multiplicative Langevin model. In this section, we study in more detail the structure of the solutions and LVs in the multiplicative stochastic model.

We have computed the characteristic LVs for the stochastic model. Figure 4 demonstrates the existence of plateaus for the differences $\eta_{n}(x, t)=h_{n}-h_{1}$, whose size decreases with $n$. The plateaus are bounded by kinks, which are prominently placed at the sites where $g_{n}(x, t)$ crosses zero [i.e., where $\left.h_{n}(x, t) \rightarrow-\infty\right]$.

The asymptotic attracting solution of Eq. (6) is the first $\mathrm{LV}, \phi(x, t)=g_{1}(x, t)$. As discussed in Sec. II C, the asymptotic solution $g_{1}(x, t)$ has the same sign everywhere. This solution is univocally determined for a given trajectory (noise realization), apart from an arbitrary nonzero constant factor. The solution $\phi(x, t)=g_{1}(x, t)$ has the statistical properties of a KPZ surface because the Hopf-Cole transformation from Eq. (6) to Eq. (7) is exact for $n=1$. In contrast, characteristic LVs for $n>1$ are saddle solutions of Eq. (6), which are forced to have regions with opposite signs. This, in turn, naturally leads to smaller growth rates $\left(\lambda_{n}<\lambda_{1}\right)$.

We find that the number of zeros of the $n$th LV is $\mathcal{N}_{0}(n)$ $=2[n / 2]$, where $[q]$ stands for the integer part of $q$. Note that Eq. (6) is not able to create new zero crossings, which implies that $\mathcal{N}_{0}(n)$ cannot fluctuate and is a conserved quantity. We also remark that $\mathcal{N}_{0}(n)$ corresponds to the number of zeros of the $n$th normal mode of the (noise-free) diffusion equation $\partial_{t} \phi=\partial_{x x} \phi$, assuming they are ordered according to their stability.

It would be very interesting to be able to write down the stochastic PDE describing the dynamics of the surface associated with the $n$th characteristic LV. However, this turns out to be a very difficult task. A more qualitative description can nonetheless be very useful. The first LV $(n=1)$ has no zeros and, as mentioned above, this allows us to exactly transform Eq. (6) into the KPZ equation (7). However for $n>1$, each $g_{n}(x, t)$ has $\mathcal{N}_{0}(n)$ zeros, which cannot be neglected when applying the Hopf-Cole transform. Indeed, one can observe that $\lambda_{n}=\left\langle\overline{\partial_{t} h_{n}}\right\rangle \neq\left\langle\overline{\left(\partial_{x} h_{n}\right)^{2}}\right\rangle$, which indicates that there must be other terms contributing to the velocity of the $n$th Lyapunov surface. As expected, the equality $\lambda_{1}=\left\langle\overline{\partial_{t} h_{1}}\right\rangle=\left\langle\overline{\left(\partial_{x} h_{1}\right)^{2}}\right\rangle$ exactly holds in the singularity-free case $n=1$.

A detailed analysis using the discrete version of Eq. (6) reveals that the stochastic PDE governing $h_{n}$ is a KPZ equation with singular (and difficult-to-treat) terms at the points where $h_{n} \rightarrow-\infty\left(g_{n} \rightarrow 0\right)$. Formally, one can expect to have

$$
\partial_{t} h_{n}=\zeta+\left(\partial_{x} h_{n}\right)^{2}+\partial_{x x} h_{n}+\sum_{i=1}^{\mathcal{N}_{0}(n)} \Xi\left[x_{i}(t)\right],
$$

where the function $\Xi\left[x_{i}(t)\right]$ accounts for singular $\delta$-like contributions at the zeros $x_{i}(t)$, whose positions move erratically around the system.

We first note that the erratic motions of the zeros [Figs. 4(b) and 4(d)] seem not to be the source of the long-ranged 
(a)

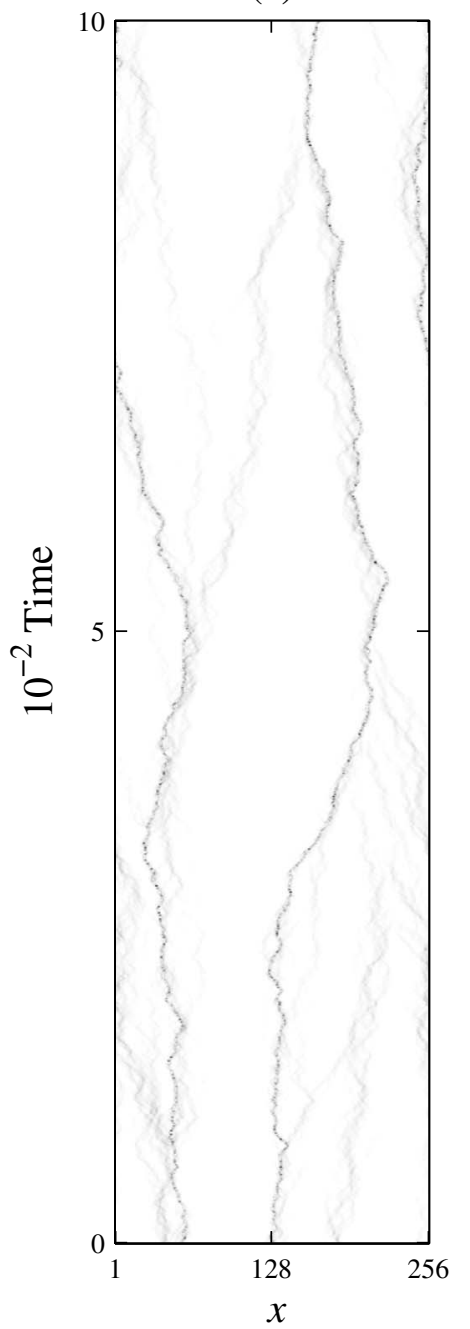

(b)

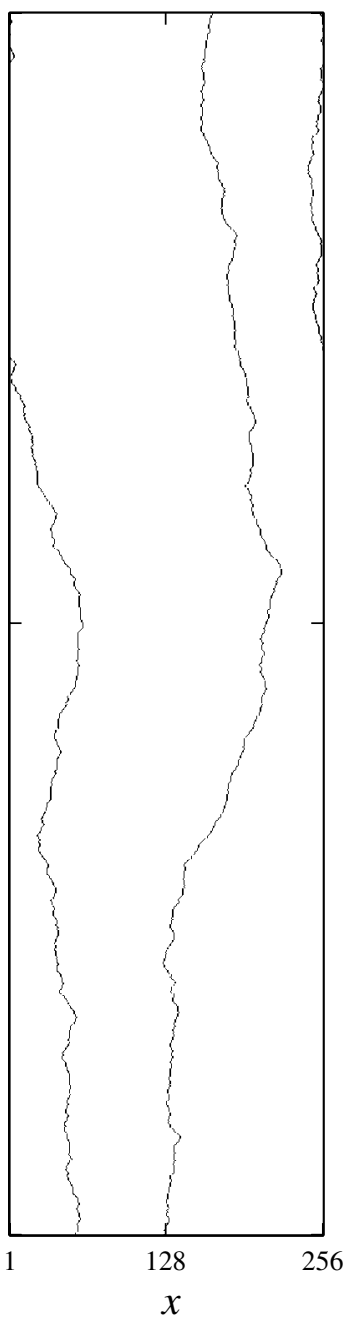

(c)

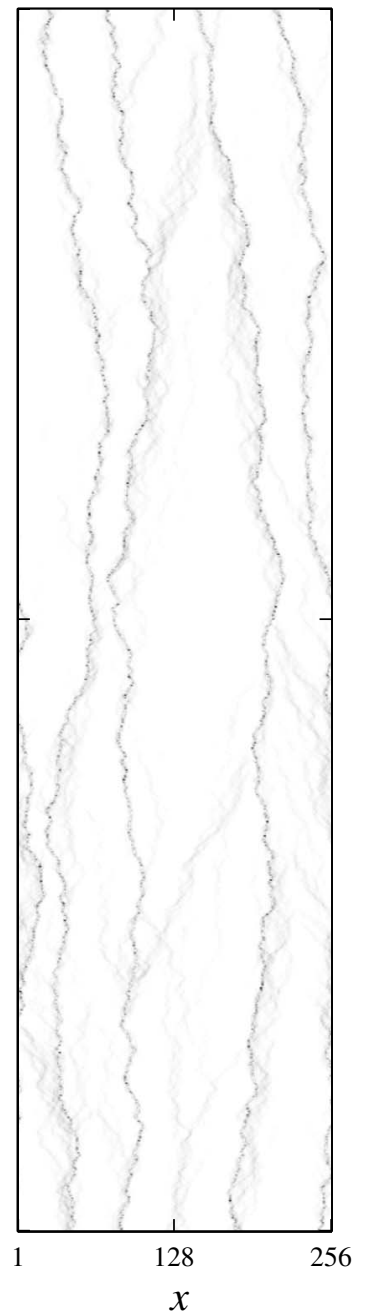

(d)

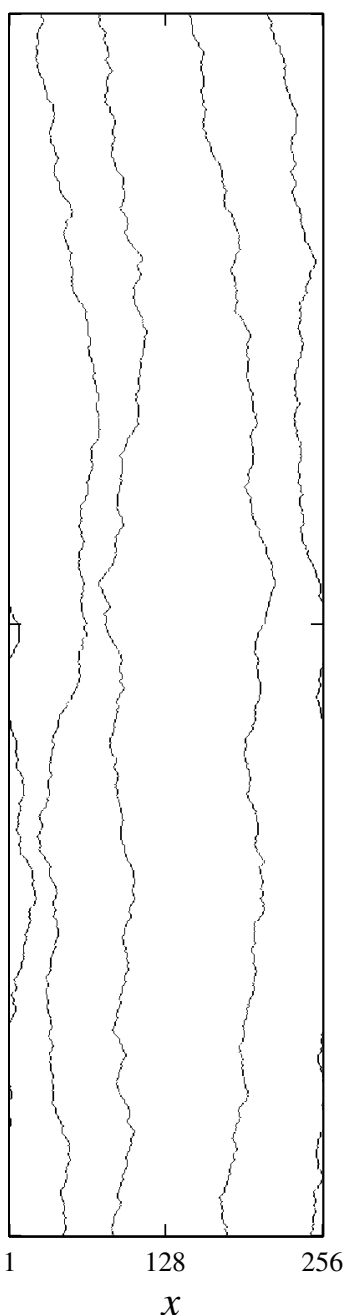

FIG. 4. Spatiotemporal plot of the fields (a) $\left|\partial_{x} \eta_{2}\right|$ and (c) $\left|\partial_{x} \eta_{4}\right|$ for the stochastic PDE (6) with $L=256$; the plateaus appear as the clear regions. The darkest regions correspond to zeros of vectors $\boldsymbol{g}_{2}$ and $\boldsymbol{g}_{4}$, indicated in panels (b) and (d), respectively. Other light gray regions in (a) and (c) correspond to kinks discussed in the text.

correlations. At long times, the erratic motion of zeros is subdiffusive: the position of the $i$ th zero satisfies $\left\langle\left[x_{i}(t)\right.\right.$ $\left.\left.-x_{i}(0)\right]^{2}\right\rangle \sim t^{\gamma}$, and we find $\gamma \approx 0.87$ for the second LV and $\gamma \approx 0.62$ for the fourth LV from numerical simulations in a system of size $L=256$. We have already shown [23] that at long wavelengths [in the $S(k) \sim k^{-1.2}$ region], surface correlations of characteristic LVs exhibit dynamic scaling. The analysis of coupled-map lattices shows a fast propagation of correlations at large scales with a dynamic exponent $z=1$ corresponding to a ballistic process $(\gamma=2 / z=2)$. Since zeros do not propagate ballistically, but subdiffusively, we conclude that information propagation at long wavelengths is mediated by a different process. The best candidates are small kinks [see, for instance, a typical kink at $x \approx 100$ in Fig. 2(f)] that can be identified (light gray traces in the plots) as traveling objects in Figs. 4(a) and 4(c). Interestingly, the dynamics of the kinks is governed by the equation of the field $\eta_{n}$, which can be written exactly inside a plateau region,

$$
\partial_{t} \eta_{n}=\left(\partial_{x} \eta_{n}\right)^{2}+\partial_{x x} \eta_{n}+2\left(\partial_{x} h_{1}\right)\left(\partial_{x} \eta_{n}\right) .
$$

The drift term, proportional to $\partial_{x} \eta_{n}$, would lead to the ballistic dynamics of the kinks with $z=1$. This provides the mechanism for the ballistic propagation of correlations observed at long wavelengths.

\section{DISCUSSION}

Our numerical results with the stochastic model (6) are particularly revealing since they explicitly show to what extent equations for growing surfaces can be used to describe STC. Specifically, Eq. (6) is invariant under multiplication by a constant, $\phi \rightarrow c \phi$, which leads to the symmetry $h \rightarrow h$ $+\ln c$ for the corresponding surface. This symmetry property leads to scale invariance of $h$ [38]. As equations for infinitesimal perturbations are always of linear type, Eq. (9), the symmetry $\phi \rightarrow c \phi$ is always fulfilled and, in turn, systems with STC will exhibit scale invariance of the associated 
Lyapunov vector surfaces. Different universality classes, depending on the existence of correlations or conserved quantities, may be obtained.

A final remark is in order. The conservation of the number of zero crossings, observed for Eq. (6), is not fulfilled in general. In a generic setting, the dynamics of perturbations would be governed by linear equations that might contain higher-order derivatives multiplied by possibly fluctuating coefficients $\xi_{i}: \partial_{t} \delta u=\xi_{1} \delta u+\xi_{2} \partial_{x} \delta u+\xi_{3} \partial_{x x} \delta u+\cdots$. Contrary to the perhaps oversimplified stochastic model (6), zeros can be created in this general situation, for instance if a drift term exists $\left(\xi_{2} \neq 0\right)$, or if $\xi_{3}$ can take negative values. Nevertheless, the scaling behavior of such a system is expected to be correctly described by Eq. (6), because those model-specific terms are actually irrelevant in the sense of the renormalization group. In conclusion, the role of zeros is very important to understand the dynamics of Eq. (6), but how they are linked to structural properties of generic systems with spatiotemporal chaos remains an issue for future work.

In summary, in this paper we have studied spatiotemporal chaos in three qualitatively different (non-Hamiltonian) systems. In all cases, characteristic (and backward) Lyapunov vectors exhibit very similar spatial structure. The $n$th HopfCole transformed LV is a piecewise copy of the first LV, with a typical plateau length that decreases with $n$. One of the three systems studied is a stochastic equation that serves as a minimal model for the leading LVs in systems whose first LV belongs to the universality class of KPZ.

\section{ACKNOWLEDGMENTS}

We thank A. Pikovsky and A. Politi for stimulating discussions. Financial support from the Ministerio de Educación y Ciencia (Spain) under projects FIS2006-12253C06-04 and CGL2007-64387/CLI is acknowledged. D.P. acknowledges support by MEC (Spain) through the Juan de la Cierva Programme.

\section{APPENDIX: COMPUTATION OF CHARACTERISTIC LYAPUNOV VECTORS}

In this appendix, we outline the procedure we have followed to obtain the characteristic Lyapunov vectors. It is based on the work by Wolfe and Samelson [26]. It assumes that that there is no degeneracy in the Lyapunov spectrum; i.e., $\lambda_{1}>\lambda_{2}>\cdots>\lambda_{L}$. For the sake of concreteness, we restrict the following discussion to the CML model (1), but it is not difficult to extend it to continuous-time systems.

Given the initial state of the system $\boldsymbol{u}\left(t_{0}=0\right)$ $=\left[u_{1}\left(t_{0}\right), u_{2}\left(t_{0}\right), \cdots, u_{L}\left(t_{0}\right)\right]$, infinitesimally small perturbations $\boldsymbol{\delta} \boldsymbol{u}\left(t_{0}\right)$ in the initial condition evolve up to linear order (i.e., in tangent space) according to

$$
\begin{aligned}
\delta u_{i}(t+1)= & \epsilon f^{\prime}\left[u_{i+1}(t)\right] \delta u_{i+1}(t)+\epsilon f^{\prime}\left[u_{i-1}(t)\right] \delta u_{i-1}(t) \\
& +(1-2 \epsilon) f^{\prime}\left[u_{i}(t)\right] \delta u_{i}(t) \equiv \sum_{j=1}^{L} T_{i j}[\boldsymbol{u}(t)] \delta u_{j}(t),
\end{aligned}
$$

with $f^{\prime}$ being the derivative of $f(y)$ with respect to $y$ and $\mathbf{T}[\boldsymbol{u}(t)]$ the $L \times L$ Jacobian matrix evaluated at $\boldsymbol{u}(t)$. The evo- lution of an infinitesimal perturbation $\delta \boldsymbol{u}\left(t_{1}\right)$ is governed by the linear equation: $\boldsymbol{\delta} \boldsymbol{u}\left(t_{0}\right)=\mathbf{M}\left(t_{0}, t_{1}\right) \boldsymbol{\delta} \boldsymbol{u}\left(t_{1}\right)$. The linear operator $\mathbf{M}$ is just the product of the Jacobian matrices evaluated along the system trajectory from $t_{1}$ to $t_{0}$, i.e.,

$$
\mathbf{M}\left(t_{0}, t_{1}\right) \equiv \mathbf{T}\left[\boldsymbol{u}\left(t_{0}-1\right)\right] \mathbf{T}\left[\boldsymbol{u}\left(t_{0}-2\right)\right] \cdots \mathbf{T}\left[\boldsymbol{u}\left(t_{1}+1\right)\right] \mathbf{T}\left[\boldsymbol{u}\left(t_{1}\right)\right]
$$

According to Oseledec's theorem [36] (details can be found in Ref. [1]), there exists the limit operator

$$
\boldsymbol{\Phi}_{b}\left(t_{0}\right)=\lim _{t_{1} \rightarrow-\infty}\left[\mathbf{M}\left(t_{0}, t_{1}\right) \mathbf{M}^{*}\left(t_{0}, t_{1}\right)\right]^{1 /\left[2\left(t_{0}-t_{1}\right)\right]}
$$

such that the logarithms of the eigenvalues are the LEs $\lambda_{n}$, and the eigenvectors form an orthonormal basis $\left\{\boldsymbol{e}_{n}\left(t_{0}\right)\right\}$. This set of eigenvectors, so-called backward LVs [28], indicates the directions of growth of perturbations grown since the remote past with exponents $\lambda_{n}$. The backward LVs are precisely the orthonormal vectors obtained using the standard Gram-Schmidt orthogonalization method to compute the LEs [37].

Conversely the directions that will grow with exponents $\lambda_{n}$ are indicated by the so-called forward Lyapunov vectors $\left\{f_{n}\left(t_{0}\right)\right\}$. They constitute an orthogonal basis formed by the eigenvectors of the operator,

$$
\boldsymbol{\Phi}_{f}\left(t_{0}\right)=\lim _{t_{2} \rightarrow \infty}\left[\mathbf{M}^{*}\left(t_{2}, t_{0}\right) \mathbf{M}\left(t_{2}, t_{0}\right)\right]^{1 /\left[2\left(t_{2}-t_{0}\right)\right]} .
$$

As with the backward LVs, the Gram-Schmidt procedure can be used to obtain forward LVs, but now going backwards in time and using the transposed Jacobian matrices because of the identity $\mathbf{M}\left(t_{2}, t_{0}\right) * \equiv \mathbf{T}^{*}\left[\boldsymbol{u}\left(t_{0}\right)\right] \mathbf{T}^{*}\left[\boldsymbol{u}\left(t_{0}+1\right)\right] \cdots \mathbf{T}^{*}\left[\boldsymbol{u}\left(t_{2}\right)\right]$. As noted by Legras and Vautard [28], the use of the transposed Jacobian (in contrast with the inverse ones) causes the forward LVs to come up with the standard ordering. This means that to obtain the first $n$ forward LVs, we need to integrate only $n$ perturbations (instead of $L-n+1$ ). Finally, note that computing forward LVs requires us to store a trajectory $\boldsymbol{u}\left(t_{0}\right), \boldsymbol{u}\left(t_{0}+1\right), \ldots, \boldsymbol{u}\left(t_{2}\right)$.

Each backward (forward) LV grows with its exponent $-\lambda_{i}$ $\left(\lambda_{i}\right)$ when it is left to evolve in the limit $t \rightarrow-\infty(t \rightarrow \infty)$. However both sets, backward and forward, do not follow their associated exponents when the time limit is reversed. For this reason, it is much more interesting to consider another set of vectors $\left\{\boldsymbol{g}_{n}(t)\right\}$, the so-called characteristic LVs, that grow with exponent $\lambda_{n}\left(-\lambda_{n}\right)$ when integrating to the far future (past),

$$
\lim _{|t| \rightarrow \infty}\left(t-t_{0}\right)^{-1} \ln \left\|\mathbf{M}\left(t, t_{0}\right) \boldsymbol{g}_{n}\left(t_{0}\right)\right\|=\lambda_{n} .
$$

The $n$th characteristic Lyapunov vector is a linear combination of the first $n$ backward Lyapunov vectors [39],

$$
\boldsymbol{g}_{n}=\sum_{i=1}^{n}\left\langle\boldsymbol{e}_{i}, \boldsymbol{g}_{n}\right\rangle \boldsymbol{e}_{i} \equiv \sum_{i=1}^{n} y_{i}^{(n)} \boldsymbol{e}_{i}
$$

$\boldsymbol{g}_{n}$ does not project on the subspace spanned by the $n-1$ first forward LVs. This allows, by means of some algebraic transformation [26], to express the coefficient vector 
$\boldsymbol{y}^{(n)}=\left(y_{1}^{(n)}, y_{2}^{(n)}, \ldots, y_{n}^{(n)}\right)$ as the one-parametric family of nontrivial solutions of

$$
\mathbf{D}^{(n)} \boldsymbol{y}^{(n)}=\mathbf{0},
$$

where the $n \times n$ matrix $\mathbf{D}^{(n)}$ is calculated using the first $n$ -1 forward LVs,

$$
D_{k j}^{(n)}=\sum_{i=1}^{n-1}\left\langle\boldsymbol{e}_{k}, \boldsymbol{f}_{i}\right\rangle\left\langle\boldsymbol{f}_{i}, \boldsymbol{e}_{j}\right\rangle .
$$

$\boldsymbol{y}^{(n)}$ is then completely determined (up to a global sign) imposing normalization of $\boldsymbol{g}_{n}$.
[1] J.-P. Eckmann and D. Ruelle, Rev. Mod. Phys. 57, 617 (1985).

[2] T. Bohr, M. H. Jensen, G. Paladin, and A. Vulpiani, Dynamical Systems Approach to Turbulence (Cambridge University Press, Cambridge, 1988).

[3] E. Ott, Chaos in Dynamical Systems (Cambridge University Press, Cambridge, 1993).

[4] H. L. Yang and G. Radons, Phys. Rev. Lett. 100, 024101 (2008).

[5] D. A. Egolf, I. V. Melnikov, W. Pesch, and R. E. Ecke, Nature 404, 733 (2000).

[6] C. Primo, M. A. Rodríguez, J. M. López, and I. Szendro, Phys. Rev. E 72, 015201(R) (2005).

[7] C. Primo, I. G. Szendro, M. A. Rodríguez, and J. M. Gutiérrez, Phys. Rev. Lett. 98, 108501 (2007).

[8] Y. Pomeau, A. Pumir, and P. Pelee, J. Stat. Phys. 37, 39 (1984).

[9] G. Giacomelli and A. Politi, Europhys. Lett. 15, 387 (1991).

[10] M. Falcioni, U. M. Marconi, and A. Vulpiani, Phys. Rev. A 44, 2263 (1991).

[11] H. Chaté, Europhys. Lett. 21, 419 (1993).

[12] A. S. Pikovsky and J. Kurths, Phys. Rev. E 49, 898 (1994).

[13] T. Taniguchi and G. P. Morriss, Phys. Rev. E 68, 046203 (2003).

[14] T. Taniguchi and G. P. Morriss, Physica A 375, 563 (2007).

[15] K. Kaneko, Physica D 23, 436 (1986).

[16] A. Pikovsky and A. Politi, Nonlinearity 11, 1049 (1998).

[17] I. G. Szendro, J. M. López, and M. A. Rodríguez, e-print arXiv:0804.0569.

[18] J. M. López, C. Primo, M. A. Rodríguez, and I. Szendro, Phys. Rev. E 70, 056224 (2004).

[19] A. D. Sánchez, J. M. López, M. A. Rodríguez, and M. A. Matías, Phys. Rev. Lett. 92, 204101 (2004).

[20] M. Kardar, G. Parisi, and Y.-C. Zhang, Phys. Rev. Lett. 56, 889 (1986)
[21] A. Pikovsky and A. Politi, Phys. Rev. E 63, 036207 (2001).

[22] C. Primo, I. G. Szendro, M. A. Rodríguez, and J. M. López, Europhys. Lett. 76, 767 (2006).

[23] I. G. Szendro, D. Pazó, M. A. Rodríguez, and J. M. López, Phys. Rev. E 76, 025202(R) (2007).

[24] G. Benettin, L. Galgani, A. Giorgilli, and J.-M. Strelcyn, Meccanica 15, 9 (1980).

[25] A. Wolf, J. B. Swift, H. L. Swinney, and J. A. Vastano, Physica D 16, 285 (1985).

[26] C. L. Wolfe and R. S. Samelson, Tellus, Ser. A 59A, 355 (2007).

[27] F. Ginelli, P. Poggi, A. Turchi, H. Chate, R. Livi, and A. Politi, Phys. Rev. Lett. 99, 130601 (2007).

[28] B. Legras and R. Vautard, in Proceedings of the Seminar on Predictability, Vol. I, ECWF Seminar, edited by T. Palmer (ECMWF, Reading, UK, 1996), pp. 135-146.

[29] A. Politi, A. Torcini, and S. Lepri, J. Phys. IV 8, Pr6 (1998).

[30] A. Trevisan and F. Pancotti, J. Atmos. Sci. 55, 390 (1998).

[31] E. N. Lorenz, in Proceedings of the Seminar on Predictability, Vol. I, ECWF Seminar, edited by T. Palmer (ECMWF, Reading, UK, 1996), pp. 1-18.

[32] E. N. Lorenz and K. A. Emanuel, J. Atmos. Sci. 55, 399 (1998).

[33] G. Boffeta, M. Cencini, M. Falcioni, and A. Vulpiani, Phys. Rep. 356, 367 (2002).

[34] V. Ahlers and A. Pikovsky, Phys. Rev. Lett. 88, 254101 (2002).

[35] M. A. Muñoz and R. Pastor-Satorras, Phys. Rev. Lett. 90, 204101 (2003).

[36] V. I. Oseledec, Trans. Mosc. Math. Soc. 19, 197 (1968).

[37] S. V. Ershov and A. B. Potapov, Physica D 118, 167 (1998).

[38] H. G. E. Hentschel, J. Phys. A 27, 2269 (1994).

[39] A component of $\boldsymbol{g}_{n}$ on $\boldsymbol{e}_{j}$ with $j>n$ would dominate the backward integration $\left(-\lambda_{j}>-\lambda_{n}\right)$. 